\title{
Bacteriophage Production Following Exposure of Lactic Streptococci to Ultraviolet Radiation
}

\author{
By BETTY E. TERZAGHI*† AND WILLIAM E. SANDINE† \\ New Zealand Dairy Research Institute, Palmerston North, New Zealand
}

(Received 2 January 1980; revised 12 August 1980)

\begin{abstract}
Single colony isolates of lactic streptococcal strains, most of which have been utilized for cheese-making in New Zealand, were u.v.-irradiated and their growth response was followed optically. All 45 strains showed either lysis or impairment of growth. Their concentrated, bacteria-free supernatants were examined by electron microscopy for the presence of phage particles. Intact phages were observed in most lysates, while disrupted phage parts were found in the remaining lysates. Plaque-forming ability was demonstrable in a few cases. Lysogenization of a different strain by one of the phages detected by this technique was attempted. Similarities and differences with respect to lytic phages which have been isolated for these same strains in New Zealand cheese factories are discussed in relation to the possible origin and control of phages.
\end{abstract}

\section{INTRODUCTION}

Control of phage infection in lactic fermentations would be of a different nature if the infecting phage were lytic in origin rather than lysogenic. Because of the common practice of using more than one strain of bacteria in starter cultures, such strain combinations are often chosen on the basis of their known phage sensitivities. However, little attention has been paid to tracing the origin of the infecting phages. We therefore wished to determine whether these phages originated as temperate phages from lysogenic starter bacteria or as lytic phages, either carried in the starter cultures in low numbers or as contaminants from external sources or both. The preceding paper (Georghiou et al., 1981) establishes that true lysogeny exists in lactic streptococci, showing that origin as a temperate phage is possible. Alternatively, it has also been demonstrated that lytic phage can be carried by lactic streptococci (Hunter, 1947; Limsowtin \& Terzaghi, 1977). Thus, both routes were suggested.

At the time of this study, the system of distribution of cheese starter cultures to most New Zealand factories was admirably suited for such an investigation into phage origins; starters were used in pairs, in rotations of four, though not all factories used the same pairs or rotations. However, all the starter cultures were dispensed from the Dairy Research Institute and whey samples were regularly returned for bacteriophage testing. Such tests established that the same phages appeared in different factories for the same starters, and that only seven morphologically distinguishable types of phages appeared although individual isolates had different host ranges (Terzaghi, 1976). We then wanted to determine which, if any, of these phages could have come from the starter bacteria themselves. Therefore, to test for lysogeny in the starter bacteria, we used a method known to induce prophages in Streptococcus cremoris (Georghiou et al., 1981) to determine the frequency of u.v.-produced phages from each of the starter cultures which had been dispensed from the Institute over the past several years. Each of these cultures had previously undergone routine dairy microbiological

† Present address: Plant Physiology Division, Department of Scientific and Industrial Research, Palmerston North, New Zealand.

† Present address: Department of Microbiology, Oregon State University, Corvallis, Oregon 97331, U.S.A. 
isolation and testing, but to minimize the possibility of a low level of lytic phage association, each culture was re-isolated using techniques shown to eliminate lytic phages from phage-carrying cultures (Limsowtin \& Terzaghi, 1977). We found that, for each strain, only after exposure to u.v. radiation were phages detectable visually by electron microscopy; in a few cases, indicator bacteria were found for these phages. Many of the phages obtained were morphologically indistinguishable from the most commonly isolated phages (Terzaghi, 1976).

\section{METHODS}

Bacteria. The following Streptococcus strains came from the New Zealand Dairy Research Institute collection: S. diacetylactis $\mathrm{DRC}_{1} ; S$. lactis $\mathrm{H}_{1}, \mathrm{ML}_{8}$ and 7962; S. cremoris $\mathrm{AM}_{1}, \mathrm{AM}_{2}, \mathrm{AM}_{3}, \mathrm{BK}_{5}, \mathrm{BR}_{4}, \mathrm{C}_{13}, \mathrm{E}_{8}, \mathrm{H}_{2}, \mathrm{~K}, \mathrm{KH}$, $\mathrm{ML}_{1}, \mathrm{ML}_{2}, \mathrm{P}_{1}, \mathrm{P}_{2}, \mathrm{R}_{1}, \mathrm{R}_{6}, \mathrm{SK}_{4}, \mathrm{SK}_{11}, \mathrm{TR}, \mathrm{US}_{3}, \mathrm{Z}_{8}, 108,114,120,130,134,158,166,168,180,186,256,266$, $286,316,368,398$ and 402 . Strain $S_{11} G$ was returned to tile Institute from a cheese factory overseas to which it had been dispatched as strain $\mathrm{SK}_{11}$. Strains $S$. cremoris $1(\mathrm{Sc} 1), \mathrm{Sc} 1-\mathrm{S}$, S. lactis $\mathrm{C}_{2}, \mathrm{C}_{2} \mathrm{Lac}^{-}$and $\mathrm{C}_{2} \mathrm{~T} 8-1$ have been described elsewhere (Huggins \& Sandine, 1977; Molskness et al., 1974).

Strain purification. All strains obtained from the Institute had previously undergone purification and testing for phage contamination as part of the routine procedure for cheese starters. We obtained these strains as vacuum-dried cultures and rehydrated them in milk. All other cultures were subcultured directly in milk. After coagulation, each culture except $\mathrm{SK}_{11} \mathrm{G}$ was again re-isolated by successive single colony isolation on $\mathrm{M} 17$ medium (Terzaghi \& Sandine, 1975) without $\mathrm{Ca}^{2+}$ supplementation, to exploit the $\mathrm{Ca}^{2+}$ requirement for phage propagation as a means of minimizing phage contamination. The cultures were then retested for activity in milk to confirm starter characteristics. All subsequent growth was on M17 medium.

Phages. Phages 601, 690,691 and 878 came from the Institute collection. They had been isolated from whey as phages lytic for strains $\mathrm{AM}_{1}$ and $\mathrm{SK}_{11}$.

Induction, electron microscopy, host range testing, transduction, phage adsorption and sucrose gradient centrifugation. The methods used have been described elsewhere (Georghiou et al., 1981; Molskness et al., 1974; Huggins \& Sandine, 1977). The u.v. source emitted $4 \mathrm{~J} \mathrm{~m}^{-2} \mathrm{~s}^{-1}$ at a distance of $29 \mathrm{~cm}$, based on coliphage T2 inactivation kinetics.

\section{RESULTS AND DISCUSSION}

\section{Induction and phage visualization}

Following repurification, all strains were u.v.-irradiated for $15 \mathrm{~s}$ after which their survival and growth responses were measured. Figure 1 shows bacterial survival, as measured by

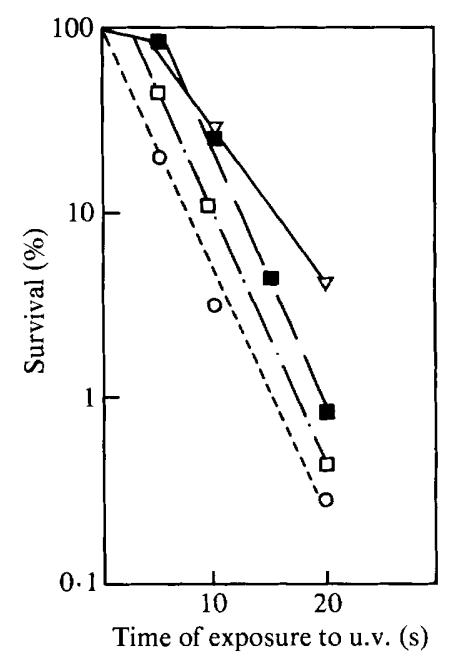

Fig. 1. Effect of increasing time of exposure to u.v. radiation on the survival of lactic streptococcal strains, as measured by colony formation on M17 medium: $O, S$. cremoris $\mathrm{ML}_{1}$ : $\square$, S. cremoris $\mathrm{P}_{2} ; \mathbf{\square}$, S. lactis $\mathrm{C}_{2} ; \nabla$, S. cremoris $\mathrm{AM}_{1}$. 
Table 1. Structural features and dimensions of phages observed after exposure of lactic streptococcal strains to u.v. radiation

\begin{tabular}{|c|c|c|}
\hline \multirow[b]{2}{*}{ Bacterial strain } & \multicolumn{2}{|c|}{$\begin{array}{l}\text { Phage dimensions } \\
\quad(\mathrm{nm}, \pm 10 \%)\end{array}$} \\
\hline & Head & Tail \\
\hline $\mathrm{DRC}_{1}$ & 49 & 100 \\
\hline $\mathrm{H}_{1}$ & 57 & $110^{*}$ \\
\hline $\mathrm{SK}_{11}^{1} \mathrm{G}$ & 57 & 110 \\
\hline $\mathrm{ML}_{8}, \mathrm{R}_{1}$ & 57 & 120 \\
\hline 7962 & 57 & $120^{*}$ \\
\hline $108,114,158$ & 57 & $135-145^{*}$ \\
\hline $\left.\begin{array}{c}\mathrm{AM}_{1}, \mathrm{AM}_{2}, \mathrm{ML}_{1}, \mathrm{SK}_{11}, \mathrm{SK}_{11} \mathrm{G} \\
\mathrm{US}_{3}, \mathrm{~K}, 134,256,266,368\end{array}\right\}$ & 57 & $135-145$ \\
\hline TR & 57 & 150 \\
\hline $\mathrm{SK}_{11} \mathrm{G}$ & 57 & 160 \\
\hline 180 & 57 & $190^{*}$ \\
\hline $\mathrm{P}_{2}, 7962$ & 57 & $220^{*}$ \\
\hline $\mathrm{C}_{2}, \mathrm{C}_{2} \mathrm{Lac}^{-}, \mathrm{C}_{2} \mathrm{~T} 8-1$ & 57 & 220 \\
\hline 186 & 57 & $230^{*}$ \\
\hline $\mathrm{BK}_{5}, \mathrm{E}_{8}$ & 57 & $240-250$ \\
\hline $\left.\begin{array}{c}\mathrm{AM}_{3}, \mathrm{P}_{1}, \mathrm{R}_{6}, \mathrm{SK}_{4}, 120,130, \\
168,398,402\end{array}\right\}$ & \multicolumn{2}{|c|}{$\begin{array}{l}\text { Intact phage seen but } \\
\text { not photographed }\end{array}$} \\
\hline $\begin{array}{l}\left.\mathrm{BR}_{4}, \mathrm{C}_{13}, \mathrm{H}_{2}, \mathrm{KH}, \mathrm{ML}_{2}, \mathrm{Z}_{8}, 166,\right\} \\
286,316, \mathrm{Scl}\end{array}$ & \multicolumn{2}{|c|}{$\begin{array}{l}\text { Only heads and/or tail } \\
\text { observed }\end{array}$} \\
\hline
\end{tabular}

* Filaments extending from the tail were observed in phages from strains $\mathbf{H}_{1}, 7962,180$ and $\mathrm{P}_{2}$ (see Fig. $2 f, h$ ). Collars were seen on phages from strains $108,114,158$ and 186.

colony-forming units, as a function of the period of exposure to u.v. radiation. Cell lysis, as determined by absorbance, was similar to that previously described for lactic streptococci (Huggins \& Sandine, 1977; Georghiou et al., 1981), namely, responses ranging from marked lysis within 2 to $3 \mathrm{~h}$ to a cessation of growth but no decrease in absorbance. The latter response resembles that reported for the relysogenized strain MU0004 of Georghiou et al. (1981), while none of the strains showed u.v.-insensitivity such as that exhibited by the prophage-cured strain MU0003.

Determination of phage induction by the criterion of plaque formation following exposure to inducing agents has been notoriously poor (Lawrence et al., 1976) and variable even on a prophage-cured strain (Terzaghi \& Terzaghi, 1978). To avoid the possibility of a misleading negative result, the lysates were examined visually for the presence of phages, rather than relying on plaque formation. Electron microscopic examination of concentrated culture supernatants showed intact particles in most lysates, and phage heads and/or tails in the remainder (Table 1; Fig. 2). This result can be compared with that of Huggins \& Sandine (1977) who found evidence of phages in $60 \%$ of the 63 strains they examined in this way. In both cases, only phages with an isometric head shape were observed and all of the New Zealand $S$. lactis and $S$. cremoris strain lysates contained phages with similar head sizes but varying tail lengths. Those observed in the Oregon State collection (Huggins \& Sandine, 1977) showed considerably more variation in head dimensions. That this is not due to differences in calibration techniques is suggested by the close agreement in particle dimensions recorded for those phages measured in both laboratories; it might, however, result from the selection of similar or related strains in New Zealand because of the criteria used for strain performance.

\section{Plaque-forming abilities}

Fourteen of the lysates were spot-tested against all bacterial strains for plaque formation. In four instances a low frequency of plaque formation was obtained. Included in this analysis 


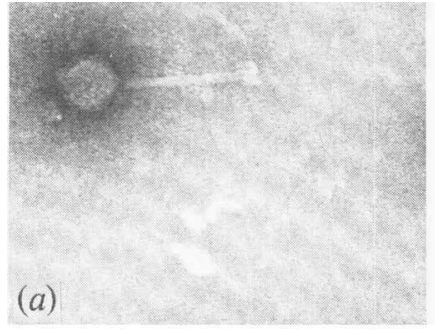

(b)

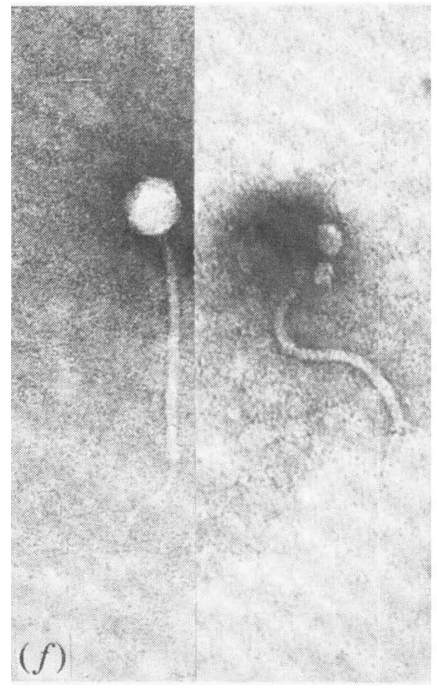

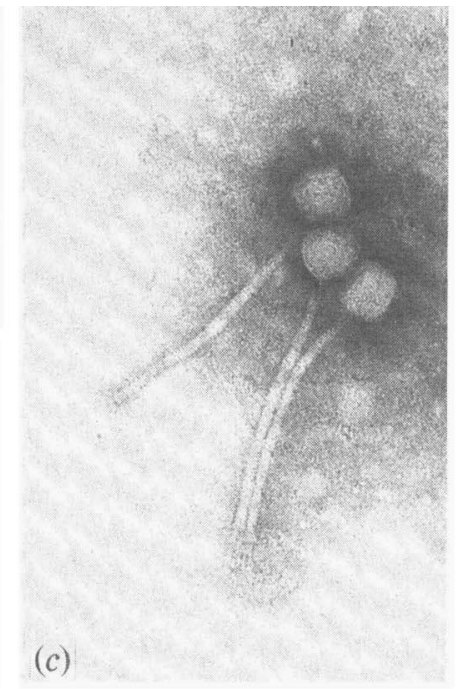
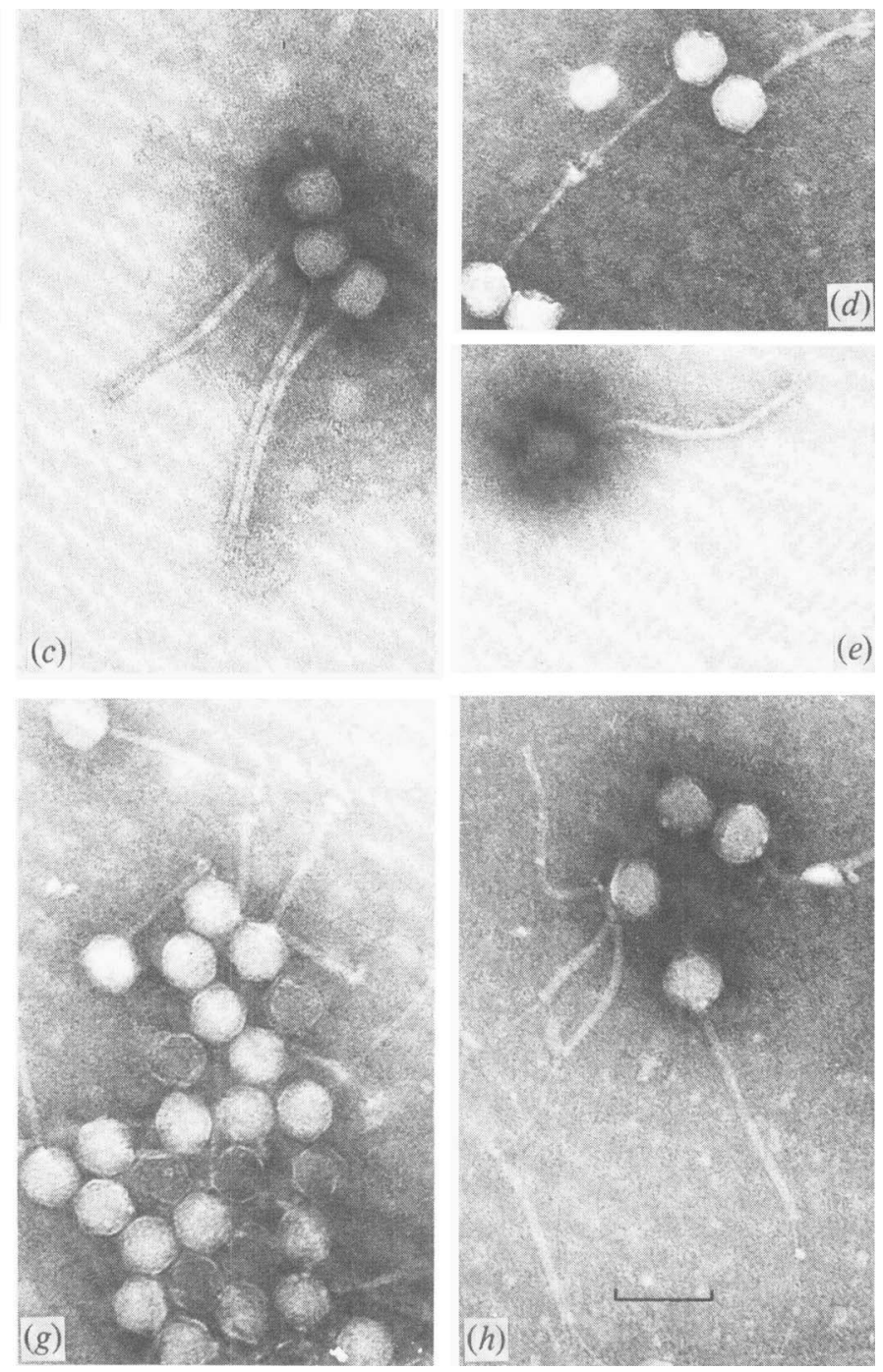

Fig. 2. Electron micrographs of phages in concentrated supernatants of lactic streptococcal strains after exposure to u.v.: (a) $S$. cremoris $\mathrm{AM}_{1} ;(b) S$. cremoris $\mathrm{AM}_{2} ;(c) S$. cremoris $\mathrm{BK}_{5} ;($ d $) S$. lactis $\mathrm{ML}_{8} ;(e) S$. cremoris $\mathrm{E}_{8} ;(f) S$. cremoris $\mathrm{P}_{2} ;(g) S$. cremoris $\mathrm{SK}_{11} \mathrm{G} ;(h) S$. lactis 7962 . Bar marker (applicable to all parts of figure) represents $100 \mathrm{~nm}$.

were strains known to serve as indicators for lytic phages attacking some of the 14 bacterial strains producing the lysates (Terzaghi, 1976). Hence, if the phages observed microscopically resulted from the presence of lytic phages in the cultures prior to induction, such phages should have been detected in this test.

Thus, by exposure to u.v. radiation, electron microscopy and (limited) plaque formation, we have shown that phages are associated with all the strains used as starter cultures in New Zealand and, by implication, with many starter strains used elsewhere in the world. Since phages were not detected as contaminants in unirradiated cultures, we conclude that the phages observed were either being produced by u.v. induction, i.e. were temperate, or if they were lytic, then the methods used for strain purification and host range testing are in need of revision. The recent report that $\mathrm{pH}$ can influence indicator ability (Terzaghi \& Terzaghi, 1978 ) illustrates the need for more research into factors affecting plating efficiency of phages. 


\section{Confirmation of lysogeny}

To further demonstrate lysogeny, attempts were made to cure strain $\mathrm{AM}_{1}$ as was done for strain MU0001 (Georghiou et al., 1981), but these were unsuccessful. Passage of bacteria through anti-phage serum could not be utilized because of the inability to propagate the presumptive induced phages on an indicator strain and, in particular, on the cured strain; thus an adequate source of pure antigen could not be obtained. However, some evidence suggesting lysogeny was obtained.

First, comparisons of phage titres (where indicator strains existed) and microscopic examination for phage particles showed that phage concentration increased with increased exposure to u.v., up to approximately $30 \mathrm{~s}$, while at the same time bacterial survival decreased (Fig. 1). This result is not easily explained by a lytic phage infection but is consistent with an increase in the number of bacteria induced up to the point where killing of the bacterial population decreased the yield.

Secondly, high frequency transducing ability is associated with temperate phages, and $\mathrm{Lac}^{+}$has been shown to be transduced at high frequency in $S$. lactis $\mathrm{C}_{2}$ (McKay et al., 1973; Molskness et al., 1974). Using our induction technique, the lysates from strains $\mathrm{C}_{2}, \mathrm{C}_{2} \mathrm{Lac}^{-}$ and $\mathrm{C}_{2} \mathrm{~T} 8-1$ were purified on sucrose gradients. In each lysate, the ensuing single band was observed to consist of morphologically indistinguishable phage particles (Table 1) which could transduce $\mathrm{Lac}^{+}$if induced from $\mathrm{C}_{2}$ or $\mathrm{C}_{2} \mathrm{~T} 8-1$ but not from $\mathrm{C}_{2} \mathrm{Lac}^{-}$.

Thirdly, if the observed phages were lytic contaminants, we would expect their morphologies to be similar to those of the seven lytic phage types. However, there are obvious discrepancies; several morphologically distinct lytic phage types isolated in New Zealand and elsewhere were not found in the lysates. In particular, lytic phages with a prolate head shape, which are common in New Zealand (Terzaghi, 1976; Limsowtin \& Terzaghi, 1976) and have a worldwide distribution (Lawrence et al., 1976), were not observed in either these inductions or those of Huggins \& Sandine (1977). Conversely, some of the phage types observed in the lysates (Fig. $2 e, f$ ) have not been isolated as lytic phages in New Zealand despite extensive use of the parent starter cultures. Thus, it appears that some phages are either incapable of lysogeny or are not u.v.-inducible while other temperate phages cannot lyse these particular starter strains.

However, there is agreement between the morphologies of the most commonly isolated lytic phages (Terzaghi, 1976) and the most frequently observed phages in u.v.-produced lysates (Fig. $2 a, b, d$ ). This may reflect the preponderance of strains related to $\mathrm{AM}_{1}$ which have been used. All of the strains related to or derived from strain $\mathrm{AM}_{1}$ had a morphologically indistinguishable phage present in their lysates; however, one derivative, strain $\mathrm{SK}_{11} \mathrm{G}$, had phages with two additional tail lengths in its lysate (Table 1; Fig. $2 \mathrm{~g}$ ). These phages were presumably acquired during factory use and their presence suggested a means for direct testing of lysogenization by transfer to the parent strain.

To confirm its relationship to $\mathrm{SK}_{11}$, the $\mathrm{SK}_{11} \mathrm{G}$ culture was phage-typed against 17 lytic phages with individual strain specificities; lysis occurred only with the one phage which also lysed the $\mathrm{SK}_{11}$ parent. To check for the presence of lytic phage, the $\mathrm{SK}_{11} \mathrm{G}$ supernatant, before and after u.v.-irradiation, was spotted on strain $\mathrm{SK}_{11}$ and the closely related strain 368 which has good indicator properties (Lowrie, 1974; Georghiou et al., 1981). Only the post-u.v. lysate produced plaques, which were clear on strain 368 but turbid on $\mathrm{SK}_{11}$, suggesting that free lytic phage were not initially present in the culture but that phage were induced which could lysogenize $\mathrm{SK}_{11}$. Accordingly, bacteria were isolated and purified from such turbid plaques and designated $\mathrm{SK}_{11}(\mathrm{Gl})$. Supernatants from these isolates did not form plaques on strain 368 , implying that they did not carry free lytic phage.

To determine if there was any identifiable difference between strains $\operatorname{SK}_{11} G$ and $\operatorname{SK}_{11}\left(G_{1}\right)$, a more sensitive phage typing was performed on both cultures against four different lytic phages attacking $\mathrm{SK}_{11}$ (Table 2). The two cultures had distinctly different phage plating abilities and, surprisingly, $\mathrm{SK}_{11}(\mathrm{G} 1)$ was more restrictive than $\mathrm{SK}_{11} \mathrm{G}$. The result with $\mathrm{SK}_{11} \mathrm{G}$ 
Table 2. Comparison of efficiency of plating of lytic phages on strains $S K_{11}, S K_{11} G$ and a putatively lysogenized derivative $S K_{11}(G 1)$

\begin{tabular}{|c|c|c|c|c|c|}
\hline \multirow[b]{2}{*}{ Phage } & \multirow[b]{2}{*}{ Morphology } & \multirow[b]{2}{*}{ Strain } & \multicolumn{3}{|c|}{ Efficiency of plating (p.f.u. $\mathrm{m}^{-1}$ ) } \\
\hline & & & $\mathrm{SK}_{11}$ & $\mathrm{SK}_{11} \mathrm{G}$ & $\mathrm{SK}_{11}(\mathrm{G} 1)$ \\
\hline 601 & Isometric & & $1.2 \times 10^{9}$ & $2 \times 10^{5}$ & $<10^{1}$ \\
\hline 690 & Prolate & & $4.7 \times 10^{8}$ & $<10^{1}$ & $<10^{1}$ \\
\hline 691 & $\begin{array}{l}\text { Isometric, } \\
\text { collar }\end{array}$ & & $7 \cdot 7 \times 10^{8}$ & $4 \times 10^{2}$ & $<10^{1}$ \\
\hline 878 & $\begin{array}{l}\text { Isometric, } \\
\text { collar }\end{array}$ & & $1.3 \times 10^{9}$ & $1.6 \times 10^{6}$ & $7 \cdot 1 \times 10^{7}$ \\
\hline
\end{tabular}

confirms the factory observation and the reason for checking the culture, namely that it was less sensitive to phage lysis than $\mathrm{SK}_{11}$. Strain $\mathrm{SK}_{11}(\mathrm{G} 1)$ was then tested for immunity to the putative lysogenizing phage. This phage, propagated on strain 368 , adsorbed to the bacteria $(>50 \%)$ yet did not form plaques, a result consistent with the expectation for lysogenic immunity. Finally, strain $\mathrm{SK}_{11}(\mathrm{G} 1)$ was u.v.-irradiated and the lysate was examined microscopically. Phages with $160 \mathrm{~nm}$ tail lengths were observed, as in $\mathrm{SK}_{11} \mathrm{G}$ (Table 1), and the lysate was tested for plaque-forming ability. Despite numerous attempts, including ageing of the indicator bacteria (Terzaghi \& Terzaghi, 1978), plaque formation did not occur on strains 368 or $\mathrm{SK}_{11}$. It is suspected that some change occurred during culturing of the indicator strains since they no longer formed plaques with the original $\mathrm{SK}_{11} \mathrm{G}$ lysate. Nevertheless, a definitive proof of lysogenization could not be given.

The results in this paper, in conjunction with other information on this defined bacteria-phage system (Terzaghi, 1976; Limsowtin \& Terzaghi, 1976, 1977; Terzaghi \& Terzaghi, 1978; Georghiou et al., 1981), suggest that the most commonly occurring lytic phages in New Zealand cheese vats originate from some of the starter strains. These phages could be induced spontaneously (Huggins \& Sandine, 1977) as well as by the heating which occurs in cheese-making. We need to know whether they then mutate to virulence or whether our plating conditions are inadequate to detect low levels of phage infection. In any case, they are normally kept in check (Limsowtin \& Terzaghi, 1976) presumably because of a combination of starter pairings and rotations (Lawrence et al., 1976), empirically determined, which make maximum use of the naturally occurring resistance and modification-restriction systems. In the case of severe infection, we need to determine whether mutations have occurred in the phage or if, by chance, unusually efficient induction or inefficient restriction has occurred because of a change in milk composition resulting in poorer bacterial growth.

With regard to phage control, we have previously shown that of the various ways in which a bacterial culture can react to a lytic phage (Limsowtin \& Terzaghi, 1977), the phage-resistant mutant can offer limited protection (Limsowtin \& Terzaghi, 1976). However, as long as bacteria are lysogenic, phages will continue to be a problem. We need to test a system using only cured strains; unfortunately, only one known cured $S$. cremoris strain exists (Georghiou et al., 1981).

These experiments point to several areas requiring research in lactic streptococci, where the lysogenic state does not conform to the Escherichia coli or Bacillus subtilis model systems. Most prominent are the need to devise new techniques for obtaining cured strains, the possibility of multiple lysogeny, an improvement in the conditions governing plaque formation, investigation of the molecules involved in phage receptor sites and whether phages with a prolate head shape are temperate. Finally, these experiments illustrate the ease with which lactic streptococcal starter cultures develop phage associations.

We thank Dr Eric Terzaghi for cooperation in this project, Loraine A. Jones and Jocelyn M. Clarke for technical assistance, the personnel in the Electron Microscope Unit of the D.S.I.R., Palmerston North for advice 
with the electron microscopy, and, finally, W.E.S. thanks the Dairy Research Institute for their hospitality during his sabbatical year.

\section{REFERENCES}

Georghiou, D., Phua, S. H. \& Terzaghi, E. (1981). Curing of a lysogenic strain of Streptococcus cremoris and characterization of the temperate bacteriophage. Journal of General Microbiology 122, 295-303.

Huggins, A. R. \& Sandine, W. E. (1977). Incidence and properties of temperate bacteriophages induced from lactic streptococci. Applied and Environmental Microbiology 33, 184-191.

Hunter, G. J. E. (1947). Phage-resistant and phagecarrying strains of lactic streptococci. Journal of Hygiene 45, 307-312.

Lawrence, R. C., Thomas, T. D. \& Terzaghi, B. E. (1976). Reviews of the progress of dairy science: cheese starters. Journal of Dairy Science 43, 141-163.

Limsowtin, G. K. Y. \& Terzaghi, B. E. (1976). Phage-resistant mutants: their selection and use in cheese factories. New Zealand Journal of Dairy Science and Technology 11, 251-256.

Limsowtin, G. K. Y. \& Terzaghi, B. E. (1977). Characterization of bacterial isolates from a phagecarrying culture of Streptococcus cremoris. New Zealand Journal of Dairy Science and Technology 12, 22-28.
LowRIE, R. J. (1974). Lysogenic strains of group N lactic streptococci. Applied Microbiology 27, 210217.

McKay, L. L., Cords, B. R. \& Baldwin, K. A. (1973). Transduction of lactose metabolism in Streptococcus lactis $\mathrm{C}_{2}$. Journal of Bacteriology 115, 810-815.

Molskness, T. A., Sandine, W. E. \& Brown, L. R. (1974). Characterization of $\mathrm{lac}^{-}$transductants of Streptococcus lactis. Applied Microbiology 28, 753-758.

Terzaghi, B. E. (1976). Morphologies and host sensitivities of lactic streptococcal phages from cheese factories. New Zealand Journal of Dairy Science and Technology 11, 155-163.

Terzaghi, B. E. \& Sandine, W. E. (1975). Improved medium for lactic streptococci and their bacteriophages. Applied Microbiology 29, 807-813.

Terzaghi, E. \& Terzaghi, B. E. (1978). Effect of lactose concentration on the efficiency of plating of bacteriophages on Streptococcus cremoris. Applied and Environmental Microbiology 35, 471-478. 